\title{
Debris Disks and Multiplicity within the 75pc Volume-limited A-Star (VAST) Survey
}

\author{
R. J. De Rosa ${ }^{1,2}$, B. Smith ${ }^{1}$, J. Bulger ${ }^{2}$, J. Patience ${ }^{1,2}$, C. Marois ${ }^{3}$,

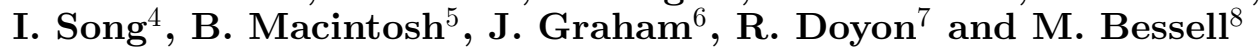 \\ ${ }^{1}$ School of Earth and Space Exploration, Arizona State University, \\ PO Box 871404, Tempe, AZ 85287-1404, USA \\ email: rjderosa@asu.edu \\ ${ }^{2}$ College of Engineering, Mathematics and Physical Sciences, University of Exeter, UK \\ ${ }^{3}$ NRC Herzberg Institute of Astrophysics, Canada \\ ${ }^{4}$ Physics and Astronomy, University of Georgia, USA \\ ${ }^{5}$ Institute of Geophysics and Planetary Physics, Lawrence Livermore National Lab, USA \\ ${ }^{6}$ Department of Astronomy, University of California at Berkeley, USA \\ ${ }^{7}$ Département de Physique, Université de Montréal, Canada \\ ${ }^{8}$ Research School of Astronomy and Astrophysics, Mount Stromlo Observatory, Australia
}

\begin{abstract}
We present the preliminary findings of an investigation of the multiplicity of debris disk stars identified within our Volume-limited A-star (VAST) multiplicity survey. Previous studies have produced conflicting results regarding the multiplicity fraction of debris disk-hosting stars compared with non-excess stars. By combining our large-scale volume-limited AO survey of A-type stars with the all-sky WISE catalogue, we have investigated the frequency of binary companions to a large sample of A-type stars with and without measured $22 \mu \mathrm{m}$ excess. The results of this study will allow for a greater understanding of the interaction between a companion star and a circumstellar debris disk, informing future study into the formation and stability of planetary-mass companions within binary systems.
\end{abstract}

Keywords. stars: circumstellar matter, stars: binaries: visual, stars: early-type

\section{Excess and control samples}

A total of 55 A-type stars within the 75 parsec sample were identified to have a significant WISE $22 \mu \mathrm{m}$ excess (Figure 1, left panel), in addition to having been searched for binaries with high-resolution adaptive optics imaging. Excess candidates were identified by comparing the observed optical and near-infrared photometry of each target to synthetic photometry derived from PHOENIX atmospheres (Hauschildt et al. 1999). Of the 55 stars within the excess sample, 15 are newly identified as candidate debris disk-hosting stars within this study. As the excess stars identified within this study were drawn from a large volume-limited sample of 636 A-type stars (De Rosa et al. 2013), we are able to construct a statistically similar control sample of 110 stars, against which the frequency of stellar companions in the excess sample can be compared (Figure 1, right panel).

\section{Binary properties of the samples}

Around the stars with measured $22 \mu \mathrm{m}$ excess, we find a multiplicity fraction of $7_{-2}^{+5} \%$, significantly lower than the $29_{-4}^{+5} \%$ measured for the control sample. The mass of each resolved companion in both samples is plotted as a function of projected separation in Figure 2. The deficiency in companions is most pronounced for separations between 

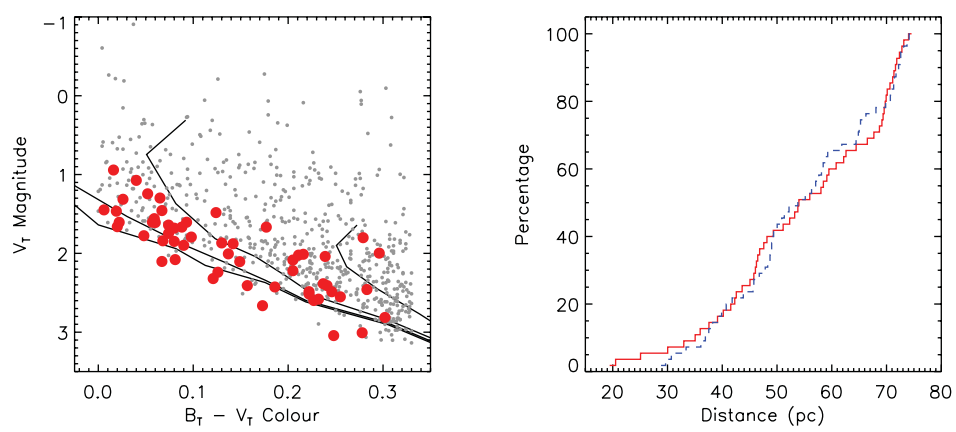

Figure 1. (left panel): A color-magnitude diagram of the 55 excess stars identified within this study (large red points), with the full VAST sample (grey points), and 100, 200, 400, and $800 \mathrm{Myr}$ isochrones (black lines; Siess et al. 2000) plotted for reference. (right panel): Cumulative distance distributions for the 55-star excess sample (red histogram), and the 110-star control sample (blue dashed histogram). The control sample was selected such that the distance distributions of the two samples were statistically similar.

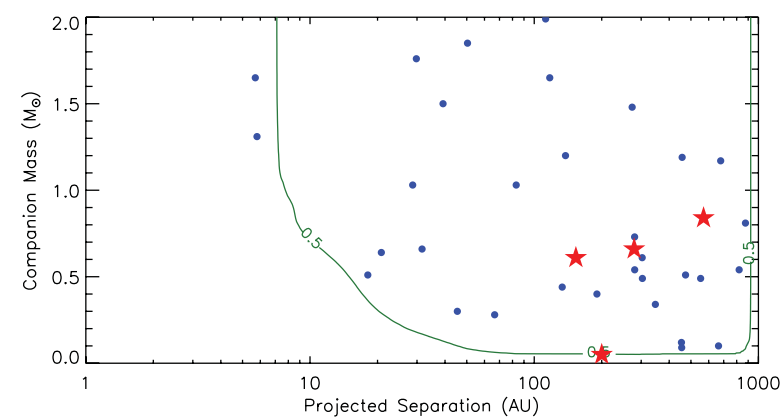

Figure 2. The separation and mass of the companions identified within the excess sample (red stars) and the control sample (blue points). The $50 \%$ sensitivity contour of the full VAST survey is plotted for reference (green curve).

10 and $100 \mathrm{AU}$, a range within which no stellar companions were resolved within the excess sample. A more detailed analysis of the sensitivity to stellar companions for both samples, as a function of projected separation, will be undertaken in order to determine the significance of this deficiency. The depressed multiplicity fraction of the excess sample is consistent with previous studies of debris disk hosting stars (e.g. Kraus et al. 2012, Rodriguez \& Zuckerman 2012). Previous studies have also shown a higher excess fraction for stars with close ( $<3$ AU) binaries (Trilling et al. 2007), a range which future extensions of this study will cover.

\section{References}

De Rosa, R. J., et al. 2013, MNRAS, submitted

Hauschildt, P. H., Allard, F., \& Baron, E. 1999, ApJ, 512, 377

Kraus, A. L., Ireland, M. J., Hillenbrand, L. A., \& Martinache, F. 2012, ApJ, 745, 19

Rodriguez, D. R. \& Zuckerman, B. 2012, ApJ, 745, 147

Siess, L., Dufour, E., \& Forestini, M. 2000, A\&A, 358, 593

Trilling, D. E., et al., 2007, ApJ, 658, 1289 